\title{
RESEARCH ON CNC TECHNOLOGY BASED ON CAD\&CAPP
}

\author{
Tao Yu', Tan Liu', Shuzhen Yang ${ }^{2}$, Wenbin Wang ${ }^{1}$ \\ ${ }^{i}$ CIMS \& Robot Center of Shanghai University, Shanghai University, China; Email: \\ alanltnew@citiz.net. ${ }^{2}$ Electromechanical Engineering Institute, Shanghai Second Polytechnic \\ University, Shanghai, China.
}

Abstract: This paper is based on a new philosophy of CNC (Computer Numerical Control), which is named as INC (Integrated Numerical Control or Intelligent Numerical Control). First it has a brief introduction of INC and describes important characteristics of INC. Second it introduces and analyses the one of INC's three key technologies: CNC technology based on CAD\&CAPP, which has mended general CNC system to fit CAD\&CAPP. It mainly includes interface technology with a STEP (Standard for the Exchange of Product Model Data) extension, embedded system and microcontroller technology, real-time Linux technology and so on. It discusses the principle and application of them also.

Key words: INC, CNC, Integrated, Intelligent.

\section{INTRODUCTION}

INC [1], proposed by Manufacturing Grid Resource Group of CIMS \& Robot Centre of Shanghai University, in a narrow sense is the abbreviation of Integrated Numerical Control. It means that abstracting some functions of traditional CIMS (Computer Integrated Manufacturing System), such as CAD, CAPP, CAM, into a series of modules, which makes the application be divided into a group of tasks frame. The tasks frames could be decomposed into detailed sub-functions. Every function module is a practical module oriented to manufacturing, such as code explaining, interpolation and PLC control. The function modules are organized and integrated into the bigger parts modules with the tasks frame. And these

This research work is financially supported by the Science and Technology Development Foundation of Shanghai Science and Technology Committee (036505008 and 05DZ11001).

Please use the following format when citing this chapter:

Yu, Tao, Liu, Tan, Yang, Shuzhen, Wang, Wenbin, 2006, in International Federation for Information Processing (IFIP), Volume 207, Knowledge Enterprise: Intelligent Strategies In Product Design, Manufacturing, and Management, eds. K. Wang, Kovacs G., Wozny M., Fang M., (Boston: Springer), pp. 492-497. 
parts modules are integrated into a concrete application $\mathrm{CNC}$ system finally with a unified user interface. The flow chart of INC is as shown in figure 1.

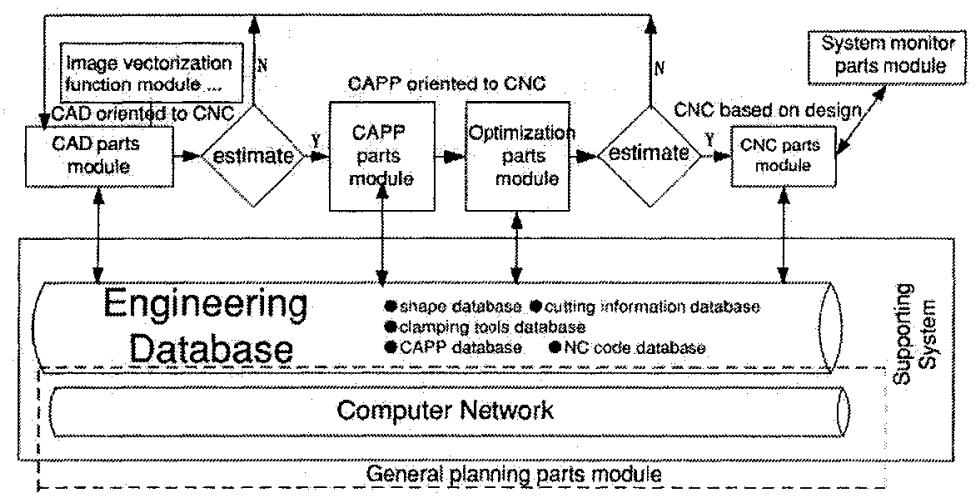

Figure 1. Flow chart of INC.

Different with some general CAD/CAPP/CAM integrated system, the focus of INC is the CNC module. Every foregoing module is the preparation of the CNC module. The other modules work for the kernel module - CNC module. For example, CAD parts module, which is oriented to $\mathrm{CNC}$ module, is not same as the general CAD system. It excludes some advanced and complex functions of computer aided design, such as three-dimensional design and three-dimensional modeling, but includes some useful functions oriented to $\mathrm{CNC}$, such as distilling of image outline, image binarization, image vectorization and so on.

In a broad sense, INC is the abbreviation of Intelligent Numerical Control. It not only has the functions of INC in a narrow sense, but also has the function of collecting system information, monitoring system conditions to do self-adapting adjustment, recording historical information to analyse and synthetize, making decision, enlarging it own expert database and etc. It has extra expert system and information collecting system than INC in a narrow sense. It also has the function of remote control.

\section{KEY POINTS OF INC}

From the concept of INC, we can see the differences between INC and DNC. DNC [2] integrates communication, control, plan and management together, maybe includes CAD, CAPP and CAM in some degree. But it aims at controlling a group of $\mathrm{NC}$ machines effective, or further controlling a whole factory. It can be seen as a kind of Distributed Manufacturing. Instead, the heart of INC is NC. It aims at manufacturing. All its work is for its last task: NC manufacturing. Its CAD parts module and its CAPP module, which 
are oriented to CNC module, are not same as the general CAD system. It has a good CSCW (Computer Supported Collaborative Work) performance and can be seen as a good application case of parallel engineering. Contrasted to Distributed Manufacturing, it is a kind of Collaborative Manufacturing. And it is a more cost-effective approach to realize CIMS than DNC. Theoretically, it can realize CAD/CAPP/CAM in a CNC machine. INC also absorbed the open attribute of ONC. For INC has adopted embedded system as its hardware platform, it is integrated and is open as ONC [3] in the same time.

Because INC is an integrated and intelligent $\mathrm{CNC}$, its key technologies not only include the technique of $\mathrm{CNC}$, but also include extra integration and intelligentization technology. And we conclude there are three kernel technologies should be considered: CAD technology oriented $\mathrm{CNC}$ (CADoCNC), CAPP technology oriented to CNC (CAPPoCNC) and CNC technology based on CAD\&CAPP (CNCbCAD\&CAPP).

In the next pages, we emphasize on discussion and study of CNCbCAD\&CAPP (CNC technology based on CAD\&CAPP) used in INC.

\section{CNC TECHNOLOGY BASED ON CAD\&CAPP}

In the traditional $\mathrm{CAD} / \mathrm{CAPP} / \mathrm{CAM}$ manufacturing process, $\mathrm{CNC}$ can only get data from CAD\&CAPP, but it can't feedback data to CAD\&CAPP. There is no two-way communication between CNC and CAD\&CAPP. For general CNC controller is made of SCM (Single Chip Micyoco), it hasn't a good extensible and configurable characteristic. Now we amend INC with newly STEP technology and embedded system technology, which is discussed as follows.

\subsection{Interface technology with a STEP extension}

In recent years, Occident has proposed and advocated a new CAM/CNC interface specification, which is named as STEP-NC [4]. STEP-NC data model is formed on the base of STEP (ISO6893) about three-dimensional geometric model. Manufacturing technology and information is added to it to substitute the traditional G-code (ISO 6893).

At present, the international standard ISO14649 comprises general principles (ISO 14649-1), universal data model (ISO 14649-10), CNC milling model (ISO 14649-11) and milling tools (ISO 14649-111), etc. The standard adopts EXPRESS (ISO 10303-11) language to define the data interface of milling and cutting operations of $\mathrm{CNC}$ in detail.

We referred its milling and turning machine part. We can express manufacturing characters such as a cutting path with a STEP extension as follows. The operating object is got by milling/cutting tool, which goes from start point to end point. And the tool may work in one depth. So the motion 
can be expressed in depth and path. The path is made up of some line segments, which are closed or not closed. The process parameter includes rotating speed basic shaft, cutting speed, and cutting path, direction (clockwise or anti-clockwise), etc. The full operation includes some special parameters, such as parts motion and rotation, arc forward and back cutting, cutter compensation, retract strategy and so on. In the lower definition, we adopt the profile style of ISO14649-10 in path description, and we adopt the approach retract strategy of ISO14649-11 in the retracting step. The following is the motion wrote in EXPRESS language [5].

ENTITY motion_feature_M

dep th: length_measure;

path_curves: p rofile;

start_point: cartesian_point;

end_point: cartesian_point;

path_direction: OPTIONAL rot_direction;

its_cutting_speed: rot_speed_measure;

rotation_direction: rot_direction;

its_feedrate: length_measure;

approach_and_lift: app roach_retract_strategy; retract_height: length_measure;

END_ENTITY

\subsection{Embedded system technology}

SCM is widely used in traditional CNC. But with the development of science and technology of computer, SCM has been found that it can not meet the requirement of more and more integrated and multifunctional and modularized CNC system. And embedded controller has been the trend of $\mathrm{CNC}$ development in the future.

Here we talk about a distinguished one of embedded systems: embedded Linux. Embedded Linux is a small type of operating system designed towards the requirement of embedded system. It comprises a kernel and some system modules customized. A small type of embedded Linux includes threes basic elements of bootloader, microkernel, and initializing process. CPU which supported embedded Linux comprises x86, Alpha, Sparc, Mips, PPC, Arm and so on. And the memory device of embedded Linux is not traditional soft disk or hard disk, but Rom, compact flash, disk on chip of Msystem or Memory stick of Sony. And the inner memory can use general memory or special RAM.

Compared to other embedded systems, Linux is not only free, but also is an open-source project. As a customizable platform Linux is supposed to be a popular system of the embedded product by many companies. Considering 
embedded Linux is open-source, good network-supporting, steady and safe and some other merits, we prefer to adopt embedded Linux as the embedded system solution in INC.

We have divided tasks to two categories: real-time task and nonreal-time task. Nonreal-time task interact with kernel through OS API (Application Programming Interface). And we use an embedded GUI between kernel and nonreal-time application to facilitate users to operate. There are three popular embedded GUI solutions in the present: Qt-embedded, Microwindows and MiniGUI. We choose MiniGUI for its small kernel and good Chinese support attributes. And for real-time tasks, they have different interplay style with kernel. We talk about it in next paragraph in detail.

\subsection{Real-time Linux technology}

The rapid development of Linux is for its applications have good compatibility of POSIX, but the architecture of Linux is a typical time-share design.

Although Linux is a single-kernel time-share OS, it has absolute advantage than other real-time embedded system for it has a better development environment and running environment. And some research organizations has developed different Linux real-time edition to make Linux used conveniently in real-time environment.

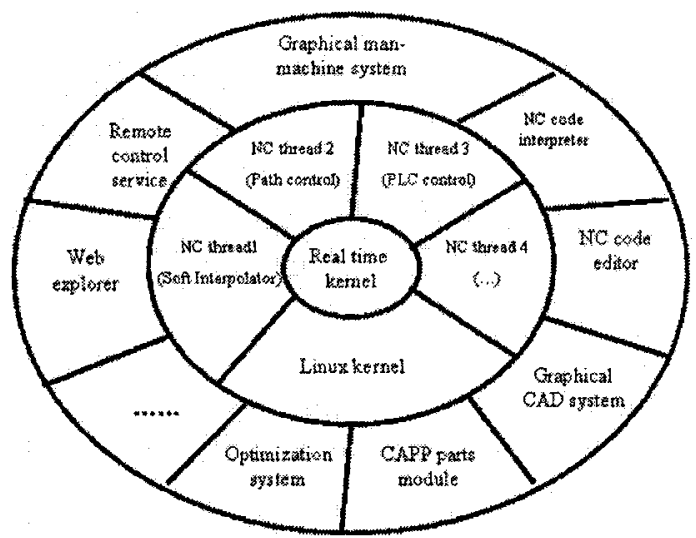

Figure 2. Software structure of $\mathrm{CNC}$ module

At the present, the real-time Linux solutions can be divided two categories. The first is to modify the original kernel adding or correcting some codes of original edition to make the kernel has a foreseeing capability. The second is to use a hybrid structure. It needs to write a new extra realtime OS kernel to handle real-time tasks. It is generally called dual-kernel architecture [6]. 
We can choose the first manner to modify the kernel. But if response time need to be less-than $1 \mathrm{~ms}$, hard real-time is recommended. We need to use the dual-kernel architecture in the condition. When using the real-time kernel, the $\mathrm{CNC}$ function modules could be divided into two layers as figure 2 .

The NC threads functions module such as PLC control and Interpolation have a high priority level, and the others have a lower priority level.

And CADbCAD\&CAPP includes some other technologies also, such as programming of device drive, kinetic control technology and so on. But they are not as distinguished as the above three, so we don't introduce them here.

\section{CONCLUSIONS AND PERSPECTIVES}

This paper proposes a new type of CNC - INC. It is a new conception in $\mathrm{CNC}$ field which gives an integrated theory oriented to manufacturing. And paper emphasizes on discussion and analysis of one of its key technology: CNC technology based on CAD\&CAPP (CNCbCAD\&CAPP).

INC is a system replacing Distributed Manufacturing with Collaborative Manufacturing that it is a novel philosophy using a cost-effective means to directly facilitate the manufacturing to enhance productivity. It is an integrated manufacturing or intelligent manufacturing.

As far as CNCbCAD\&CAPP is concerned, it solves the problem how to setup a two-way communication between $\mathrm{CNC}$ and CAD\&CAPP. And it gives a solution using embedded system and real-time Linux kernel to substitute traditional PC or SCM to make INC have a unified external standard controller interface and have extensible and configurable modules.

\section{REFERENCES}

1. Tan Liu, Tao Yu, Shuzhen Yang and Wenbin Wang, "INC: A New Philosophy of CNC", Proceedings of 2005 International Conference on Machine Learning and Cybernetics, Guangzhou, China, August. 17-21, 2005, pp 1338-1344

2. P. Stout, and R. Leonard,"The introduction of DNC technology as a partial approach to achieving the objectives of CIM", Computer-Aided Engineering Journal, China, 1999.2, pp. 17-20.

3. Yulong Wang, "Open architecture for $\mathrm{CNC}$ controller and their developing prospect", $C N C$ \& Software, China, 2004.4, pp. 75-76

4. Official STEP-NC website: www.step-nc.org

5. Weiyue Xiao, Intelligent Information Porcessing Technology in CAPP, National Science Publisher, Changsha, China, 2002.

6. Kanfu Wang, Technological Base of Numerical Control, Machinery Industry Press, Beijing, China, 2001.4 